\section{Experimental Demonstration of Cross-Channel Interference Cancellation for Digital Filter Multiple Access PONs} \\ Giddings, Roger P.; Duan, Xiao; Tang, Jianming \\ DOI: \\ 10.1364/OFC.2016.Th3C.5
}

Published: 23/03/2016

Peer reviewed version

Cyswllt i'r cyhoeddiad / Link to publication

Dyfyniad o'r fersiwn a gyhoeddwyd / Citation for published version (APA):

Giddings, R. P., Duan, X., \& Tang, J. (2016). Experimental Demonstration of Cross-Channel Interference Cancellation for Digital Filter Multiple Access PONs. Paper presented at Optical Fiber Communication Conference, Anaheim, United States.

https://doi.org/10.1364/OFC.2016.Th3C.5

\footnotetext{
Hawliau Cyffredinol / General rights

Copyright and moral rights for the publications made accessible in the public portal are retained by the authors and/or other copyright owners and it is a condition of accessing publications that users recognise and abide by the legal requirements associated with these rights.

- Users may download and print one copy of any publication from the public portal for the purpose of private study or research.

- You may not further distribute the material or use it for any profit-making activity or commercial gain

- You may freely distribute the URL identifying the publication in the public portal ?
}

Take down policy

If you believe that this document breaches copyright please contact us providing details, and we will remove access to the work immediately and investigate your claim. 


\title{
Experimental Demonstration of Cross-Channel Interference Cancellation for Digital Filter Multiple Access PONs
}

\author{
R.P. Giddings, X. Duan and J.M. Tang \\ School of Electronic Engineering, Bangor University, Bangor, LL57 1UT, UK
}

\begin{abstract}
A DSP-based cross-channel interference cancellation technique with initial conditionfree fast convergence is proposed and experimentally demonstrated, for the first time, which improves BER performances up to 1000 times after only one iteration in IMDD DFMA-PONs. OCIS codes: (060.0060) Fiber optics and optical communications; (060.4230) Multiplexing.
\end{abstract}

\section{Introduction}

A digital filter multiple access (DFMA) technique [1] has been recently proposed to utilise centralized SDN controller-managed, dynamic software-reconfigurable, embedded digital orthogonal filters to considerably enhance the functionality, flexibility and upgradability of both DSP-based optical transceivers and network operations. Furthermore, when combined with optical filter- and E-O-E conversion-free, cost-effective (ROADMs) [2] that perform the add/drop operations at wavelength, sub-wavelength and orthogonal sub-band levels, DFMA also has great potential for supporting future cloud access networks capable of seamlessly integrating traditional optical access networks, metropolitan area optical networks and mobile front-haul/back-haul networks, as, apart from the features mentioned above, DFMA also has other salient advantages including [1]: i) Fully supporting the SDN solution with the network control further extended to the physical layer; ii) Providing good compatibility with all existing networks; iii) Transparent to both underlying transmission technologies and network topologies and iv) Enhanced data security.

In [1], DFMA has been applied in cost-sensitive PON application scenarios, and detailed ONU count-dependent performance characteristics of IMDD DFMA-PONs have been numerically explored in terms of maximum achievable transmission capacity and system power budget [1]. In addition, a real-time OFDM-based IMDD DFMA-PON system has also been experimentally demonstrated over a $25 \mathrm{~km}$ SSMF link [3]. The aforementioned investigations [1,3] have shown that DFMA-PON performance characteristics may be considerably degraded when imperfect orthogonality between various information-bearing digital filters occur because of a diversified range of nonlinearities associated with signal generation, transmission, channel routing/switching and detection. To address such a technical challenge, two general DSP-based techniques may be considered including strong channel equalization such as least mean square (LMS)-based equalization [4] and successive interference cancellation [5]. However, when applied to DFMA-PONs, these two techniques suffer from a number of significant disadvantages outlined below: i) signal modulation format-dependent performance; ii) high DSP complexity which increases with higher modulation formats; iii) a large number of iterations required to achieve the performance convergence, and iv) high convergence sensitivity to initial conditions.

In this paper, we propose and experimentally demonstrate, for the first time, a simple but highly effective DSPbased cross-channel interference cancellation (CCIC) technique, which operates by estimating and subsequently cancelling the cross-channel interference (CCI) effect associated with IMDD DFMA-PON systems. The proposed CCIC technique is modulation format-independent, has comparatively low DSP complexity, has a fast iteration speed and it is completely free from dependence on any initial conditions. The results presented in this paper suggest that the CCIC technique can be highly advantageous when applied to IMDD DFMA-PONs.

\section{CCIC principle}

For simplicity but without losing generality, a two-channel IMDD DFMA-PON system carrying two independent information signals is shown in Fig. 1. The system employs a pair of shaping (matching) filters in the transmitter (receiver) which form a Hilbert pair to produce channel orthogonality, thus these two signals occupy the same spectral region [1]. A full description of the digital orthogonal filter-enabled channel multiplexing/demultiplexing principle is given in [6]. As the CCI occurs mainly between two spectrally-overlapped orthogonal channels, the considered two-channel system is thus sufficient to evaluate the CCIC technique. In addition, an OFDM signal is considered for each channel in Fig.1, the DFMA technique can, however, support arbitrary signal modulation formats. The top-level DSP functional blocks illustrated in Fig. 1 includes the proposed CCIC block in the receiver.

In the CCIC technique applied here we mitigate the linear CCI generated as a consequence of the non-ideal physical system frequency response. A channel's received signal can thus be considered as a linear combination of the desired signal and the CCI from its associated orthogonal channel. If an estimate of the linear CCI on a channel 


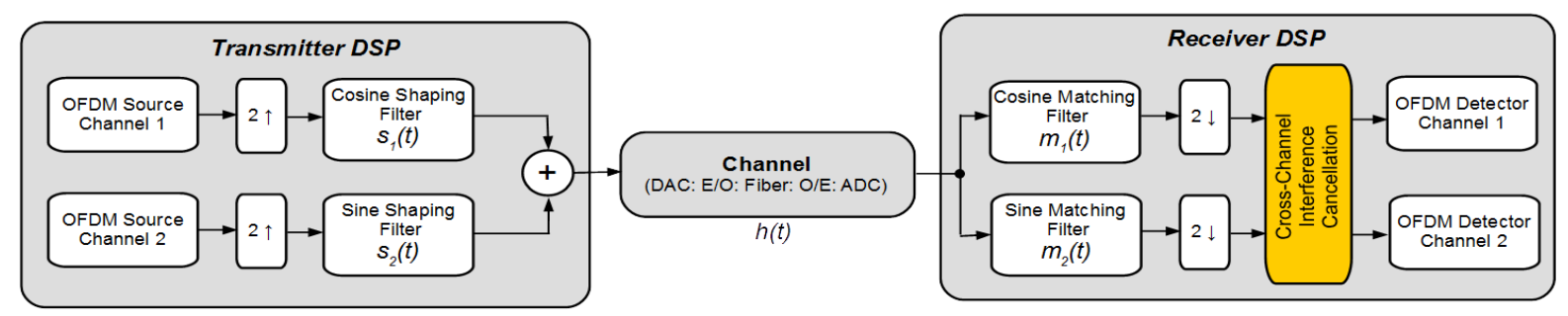

Fig 1. Two-channel DFMA-PON system carrying OFDM signals on each channel.

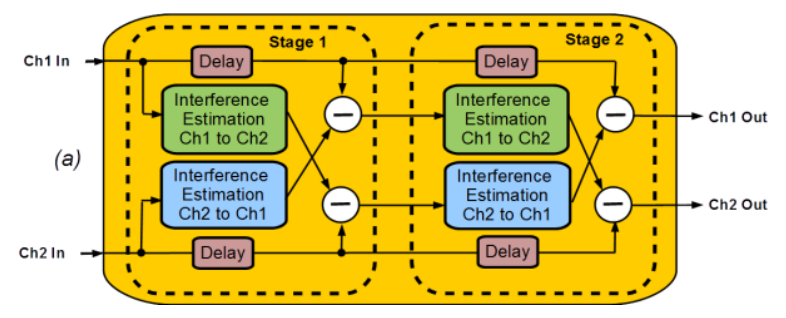

(b)

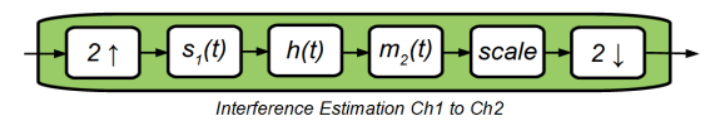

(c)

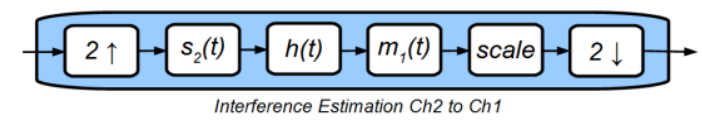

Fig 2. (a) 2 stage Cross-Channel Interference Cancellation function. Interference estimation functions: (b) Ch1 to Ch2 and (c) Ch2 to Ch1

can be determined it can then be subtracted from the channel's received signal to give an improved estimate of the transmitted signal. The CCI signals are functions of the transmitted signal, however as these are clearly not available at the receiver, the recovered signals, before signal demodulation, are used as the best estimate of the transmitted signals for input to the CCIC function. The first CCIC stage continuously computes the two CCI estimates and subtracts these from the suitably delayed received signals, thus generating improved estimates of the transmitted signals. Further CCIC stages can then be used to implement successive CCIC iterations to iteratively improve the accuracy of the transmitted signal estimates. The internal DSP structure of the evaluated CCIC block is shown in Fig. 2(a), which shows two successive CCIC stages, the offline DSP being easily modified to implement either one, two or more iterations for evaluating the optimum iteration requirement. The DSP processes that calculate the CCI estimates operate by emulating the real processes that generates the actual CCI. For ch1 to ch2 CCI this process starts by $2 \mathrm{x}$ up-sampling, is followed by 3 distinct filter stages of; transmitter shaping filter $s_{l}(t)$, the entire physical channel $h(t)$ and the receiver matching filter $m_{2}(t)$ and ends with $2 \mathrm{x}$ down-sampling. Here it is the physical channel $h(t)$ that generates the CCI leakage from ch1 (cosine) to ch2 (sine) which cannot be removed by the sine-channel selective matched filter on ch2. The interference estimation function for ch1 to ch2 is therefore as shown in Fig. 2(b). The corresponding ch2 to ch1 interference estimation function is shown in Fig. 2(c).

\section{Experimental system setup}

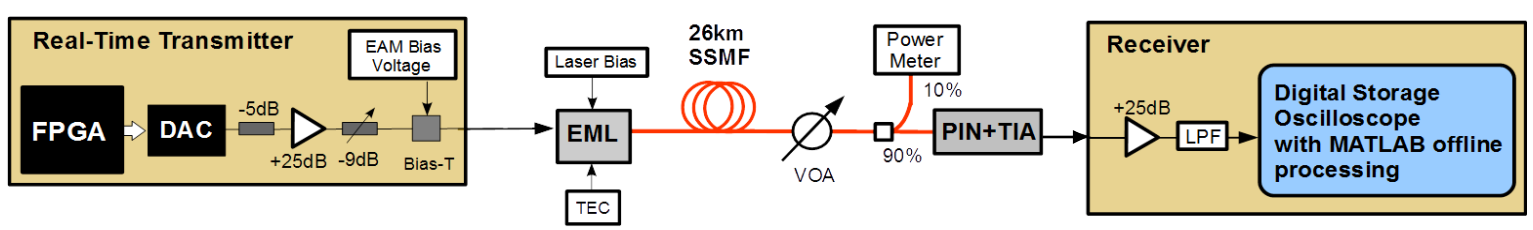

LPF: LoW Pass Filter VOA: Variable Optical Attenuator PIN+TIA: Photodetector with integrated Transimpedance Amplifer EML: Electro-absorption Modulated Laser

Fig 3. Experimental system setup for point-to-point 2 channel system with transmission over $26 \mathrm{~km} \mathrm{SSMF}$

The experimental system setup is shown in Fig.3. The transmitter is implemented in real-time using an FPGA and DAC operating at 2GS/s [3]. An IMDD optical link is employed with an EML as an optical intensity modulator, a fiber link of $26 \mathrm{~km}$ SSMF and a direct-detect photodetector consisting of integrated PIN and TIA. The key parameters of the OFDM signals on each channel and the optical link are given in Table 1. The receiver is implemented with a digital storage oscilloscope (DSO) which captures a sequence of digital samples and applies offline DSP using MATLAB. The DSO captures samples at $25 \mathrm{GS} / \mathrm{s}$ which are resampled to $2 \mathrm{GS} / \mathrm{s}$ in the first stage of the offline DSP. As performance is sensitive to the receiver sampling timing offset, the optimum timing offset is determined when resampling from 25 to $2 \mathrm{GS} / \mathrm{s}$. The offline DSP then performs all the receiver DSP functions as illustrated in Fig. 1 and 2, and is modified appropriately for the desired number of CCIC iterations. All digital filters in both the real-time transmitter and offline receiver are implemented as 32-tap FIR filters [3]. 
Table I

\begin{tabular}{cccc}
\hline Parameter & Value & Parameter & Value \\
\hline Total number of IFFT/FFT points & 32 & Oscilloscope sample rate [re-sampled rate] & $25 \mathrm{GS} / \mathrm{s}$ [2GS/s] \\
Data carrying subcarriers & 13 per channel & Raw data rate per channel [aggregate] & $1.625 \mathrm{~Gb} / \mathrm{s}[3.25 \mathrm{~Gb} / \mathrm{s}$ ] \\
n-th subcarrier frequency & $n \times 31.25 \mathrm{MHz}$ & EML wavelength / output power & $1550 \mathrm{~nm} / 2.5 \mathrm{dBm}$ \\
Modulation format on all subcarriers & $16-\mathrm{QAM}$ & EML laser bias current & $125 \mathrm{~mA}$ \\
OFDM symbol rate & $25 \mathrm{MHz}$ & 3-dB EML modulation bandwidth & $10 \mathrm{GHz}$ \\
Cyclic prefix & $25 \%$ & EAM bias voltage / driving voltage & $-0.7 \mathrm{~V} / 2 \mathrm{Vpp}$ \\
DAC sample rate & $2 \mathrm{GS} / \mathrm{s}$ & PIN detector bandwidth / sensitivity & $12.4 \mathrm{GHz} /-19 \mathrm{dBm} *$ \\
DAC resolution & $8 \mathrm{bits}$ & & \\
\hline "Subcarriers 1 and 2 are dropped & & $*$ Corresponding to $10 \mathrm{~Gb} / \mathrm{s} \mathrm{NRZ} \mathrm{data} \mathrm{at} \mathrm{a} \mathrm{BER} \mathrm{of} 1.0 \times 10^{-9}$
\end{tabular}

\section{Experimental results}

Fig. 4 (a) and (b) show the measured subcarrier BERs versus subcarrier index for channel 1 and channel 2 , respectively, and their corresponding total channel BERs versus received optical power are also presented in Fig. 5 (a) and (b). In all these figures, BER performances are plotted for the cases of no CCIC and including the CCIC technique with one iteration and two iterations. It is evident that, when no CCIC is applied, the system frequency response roll-off of $\sim 11 \mathrm{~dB}[3]$ results in unacceptably high subcarrier and total channel BERs. To the sharp contrary, the BER performances after only one CCIC iteration are seen to be drastically improved by a factor as high as 1000 , and the transmission system can now meet the typical hard-FEC limit. As a direct result of the proposed CCIC technique's powerfulness, the second CCIC iteration is not necessary, as shown in Fig.4 and Fig.5. This indicates that the CCIC technique has a very fast iteration speed. In addition, from these figures it can also be seen that the CCIC technique is almost equally effective for various subcarriers experiencing a frequency response roll-off difference as large as $11 \mathrm{~dB}$, and also is effective for a received optical power variation range as large as $4 \mathrm{~dB}$.
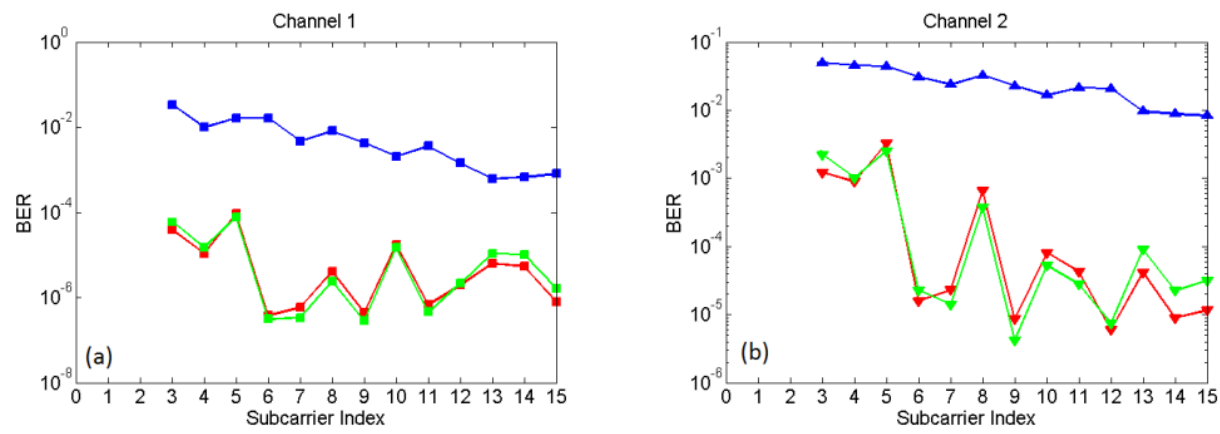

Fig 4. BER vs subcarrier index for transmission over 26km SSMF. (a) Channel 1 (b) Channel 2. Blue: Without CCIC, Red: 1st CCIC iteration, Green: 2nd CCIC iteration
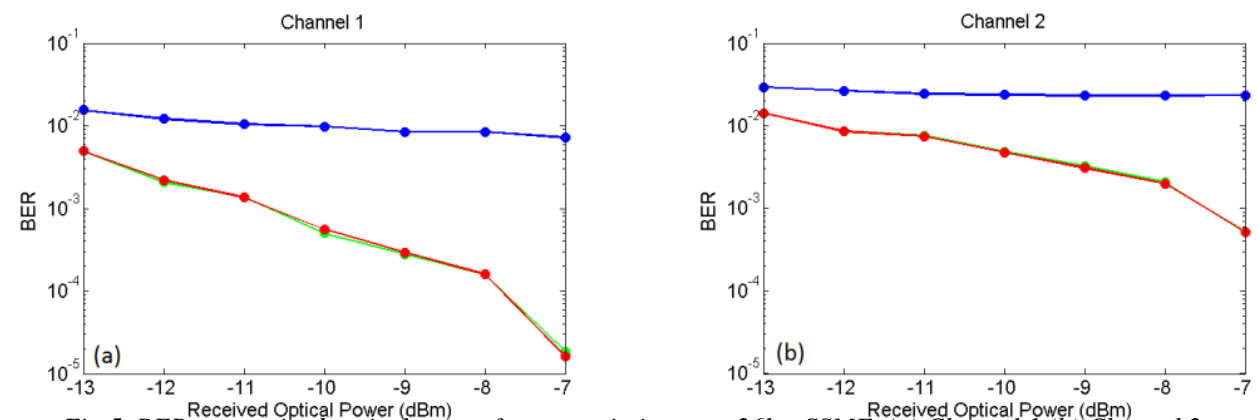

Fig 5. BER vs. received optical power for transmission over $26 \mathrm{~km}$ SSMF. (a) Channel 1 (b) Channel 2. Blue: Without CCIC, Red: 1st CCIC iteration, Green: 2nd CCIC iteration

\section{Conclusions}

A DSP-based, initial condition-free and fast convergence CCIC technique has been proposed and experimentally demonstrated, which is shown to be a highly effective in mitigating the CCI effect. Experimental results have shown that one CCIC iteration is capable of improving BER performances by up to 1000 times in IMDD DFMA-PONs.

\section{References}

[1] M. Bolea, et.al., J. Opt. Comm. Netw 7(4), 215, 2015.

[3] X. Duan, et.al., Opt. Express, 22(16), 19674, 2014.

[5]A.L.C. Hui et. Al., IEEE Tran. Comm, 46(3), 384,1998.

[2] W. Jin, et.al., J. Light. Technol., 33(19), 4124, 2015.

[4] L. Tao, et al., IEEE Photon. Tech. Let., 25(23), 2346, 2013.

[6] M. Bolea, et.al., J. Light. Technol., 32(6), 1200, 2014. 